\title{
KDM5C Gene
}

National Cancer Institute

\section{Source}

National Cancer Institute. KDM5C Gene. NCI Thesaurus. Code C97598.

This gene is involved in histone modification. 